\title{
microenvironnement tumoral
}

> Les tumeurs se développent au sein d'un microenvironnement cellulaire et tissulaire complexe. Celui-cin'est pas inerte ; ses composants influencent l'évolution de la maladie, certains l'accélèrent, d'autres la freinent. Dès les premières étapes de la transition d'un tissu normal à un tissu dysplasique, puis tumoral bénin, puis tumoral malin et enfin jusqu'aux métastases, les interactions potentielles entre les cellules transformées et leur microenvironnement orientent l'évolution de ces processus. Un tissu siège d'une inflammation chronique favorise l'apparition de tumeurs, lesquelles, par les facteurs de néoangiogenèse, d'immunosuppression et d'inflammation qu'elles produisent, renforcent les propriétés nourricières et énergétiques de leur microenvironnement. À l'opposé, la présence d'une forte réaction immunitaire de type Thl/cytotoxique mémoire peut prévenir la transition de tumeurs bénignes vers des cancers et contrôler la croissance et l'invasivité des cellules tumorales même au stade métastatique. La connaissance de ces multiples phénomènes, décrits dans les différents articles de ce numéro thématique de médecine/sciences, a un impact clinique majeur, puisque de nouveaux tests pronostiques et des approches thérapeutiques efficaces ciblant le microenvironnement tumoral sont aujourd'hui en plein développement. <

\section{Le microenvironnement tumoral : partenaire} à part entière du développement tumoral

Le cancer représente une des menaces majeures pour la santé de l'homme. Avec plus de dix millions de nouveaux cas et plus de cinq millions de morts par an, il est la première cause de mortalité dans de nombreux pays. Résultant de la dérégulation des mécanismes

Cet article fait partie du numéro thématique publié par médecine/sciences en avril 2014 et intitulé « Microenvironnement tumoral ».

\section{Matrice nourricière, champ de bataille et cible thérapeutique des cancers}
Wolf H. Fridman ${ }^{1,2}$, Catherine Sautès-Fridman ${ }^{1,2}$

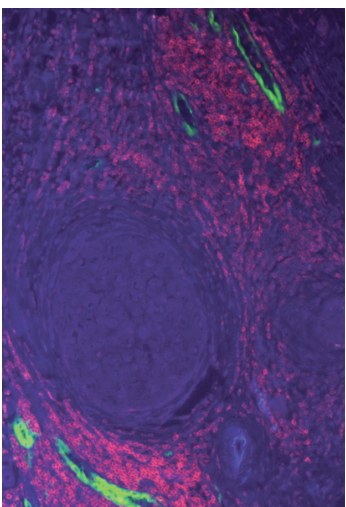
${ }^{1}$ Centre de recherche des Cordeliers, Inserm UMRS 872, 15, rue de l'École de Médecine, 75006 Paris, France ;
${ }^{2}$ Université Paris-Descartes, Paris, France.
herve.fridman@crc.jussieu.fr

de prolifération, de survie et de différenciation cellulaires, le développement de cellules potentiellement cancéreuses est inhérent à l'existence même de la vie. Il est donc vraisemblable que l'éradication complète des cancers est illusoire. Cependant, la comparaison des caryotypes de cellules cancéreuses à leur équivalent normal révèle de multiples anomalies, et c'est l'un des reflets des modifications génétiques de tous ordres qui caractérisent les cellules cancéreuses. Ces anomalies s'accumulent au cours d'un long processus qui permet successivement la transition d'une cellule normale à une cellule «potentiellement » cancéreuse ou précancéreuse, la formation d'une tumeur primitive localisée, l'invasion tissulaire et enfin le détachement de cellules cancéreuses de la tumeur primitive et leur intravasation, ce qui permet leur migration puis la formation de métastases à distance. La connaissance des processus qui aboutissent à la transformation maligne, au passage d'une tumeur précancéreuse à un cancer invasif et au développement de métastases permet de mettre en place de nouvelles approches de prévention et de thérapie visant à diminuer l'incidence, la morbidité et la mortalité des cancers.

L'élément initial de la transformation d'une cellule normale, souvent épithéliale, en cellule potentiellement cancéreuse est l'acquisition d'une autonomie vis-à-vis des contrôles de la prolifération cellulaire, qui aboutit à la multiplication anarchique de cette cellule. L'acquisition de défauts dans les mécanismes naturels d'apoptose, qui permettent de contrôler le nombre de cellules d'un tissu et d'en éliminer des cellules anormales, est aussi impliquée. Ces modifications résultent souvent de mutations somatiques dans les gènes contrôlant la prolifération et la survie cellulaires (oncogènes et gènes 
suppresseurs de tumeurs). De multiples autres remaniements génétiques, - mutations, amplifications, fusions ou délétions de gènes - et épigénétiques, - méthylations, épissages différents d'ARN, surproduction de protéines, modifications de glycosylation et de lipides, permettront une invasion tumorale locale, la transition épithéliomésenchymateuse et le détachement des cellules de la tumeur primitive pour former des métastases à distance [13] $(\rightarrow)$ par les voies sanguines, lymphatiques, sympathiques et parasympathiques. $\rightarrow$ Voir la Synthèse de S. Hubert et J.P. Abastado, page 378 de ce numéro
Durant toutes ces étapes, les cellules tumorales interagissent avec les éléments structuraux (matrice extracellulaire), cellulaires, dont les fibroblastes $[14,15](\rightarrow)$, et moléculaires du tissu qui les enserre. Les éléments forment le microenvironnement tumoral, qui est très hétérogène et différent selon l'organe où naît $(\rightarrow)$ Voir les Synthèses de $\varepsilon$. Buache et al., et de J. Albrengues et al., pages 385 et 391

de ce numéro et se développent la tumeur primitive et ses métastases. Cet environnement évolue conjointement à l'évolution du cancer et à l'invasion, qui sont elles-mêmes dépendantes du microenvironnement tumoral. II est désormais clairement établi que le cancer ne résulte pas seulement d'une série d'événements oncogénétiques, mais que le microenvironnement est un partenaire à part entière et exerce un impact important sur l'évolution de la maladie. Ainsi, la progression et l'impact clinique d'une tumeur dépendent de la dynamique d'interactions entre les cellules malignes et leur microenvironnement. Comme le montrent les articles de ce numéro thématique de médecine/sciences, la majorité des éléments du microenvironnement favorisent le développement des tumeurs, et quelques uns le contraignent. Malgré la diversité des tissus et des cellules qui composent ce microenvironnement, un certain nombre de règles sont aujourd'hui établies, et leur application fournit des outils de compréhension, mais aussi de prévention, de pronostic et de traitement des cancers. Le présent numéro de médecine/sciences aborde les aspects essentiels des interactions entre les cellules tumorales et les diverses composantes de leur microenvironnement. II met en place le rôle potentiel de ces différentes composantes aux différentes étapes du développement des cancers.

\section{L'inflammation chronique, facteur favorisant et matrice nourricière}

De nombreuses études épidémiologiques ont établi que l'exposition à certains agents chimiques ou physiques, ou à des agents infectieux, favorisait l'émergence de cancers. Parmi ces facteurs environnementaux, certains agissent directement sur la transformation maligne en modifiant I'ADN des cellules cibles par cassure (irradiation, UV), par adduits (tabac) ou en introduisant des oncogènes (certains papilloma virus [HPV16 ou HPV18] ou virus herpes [EBV, Epstein Barr virus]). Cependant, les facteurs environnementaux ont aussi une action sur le microenvironnement tumoral. L'exemple le plus démonstratif est celui de la bactérie Helicobacter pylori, qui induit un cancer de l'estomac non pas par transformation maligne, mais par l'induction d'une inflammation chronique accompagnée d'une forte production gastroduodénale de cytokines pro-inflammatoires (IL [interleukine]l,
IL6, TNF $\alpha$ [tumor necrosis factor $\alpha$ ]), qui sont aussi des facteurs de croissance pour les cellules épithéliales. Parallèlement, les macrophages recrutés au site inflammatoire produisent des agents augmentant la néovascularisation et la perméabilité vasculaire, apportant nutriments et oxygène à une tumeur naissante. Des études épidémiologiques ont montré qu'une antibiothérapie appropriée peut être un moyen de prévention du développement d'un cancer de l'estomac. De nombreux agents biologiques, physiques ou chimiques, présentés dans le Tableau I, favorisent l'émergence d'un cancer par un mécanisme similaire. L'inflammation chronique «formant le lit du cancer», d'autres états pathologiques capables de provoquer ce type d'inflammation sont aussi des facteurs favorisants. Au premier rang se trouve l'obésité qui, par la modification du métabolisme, mais aussi par la création d'une inflammation adipeuse, est un facteur de risque majeur. Ainsi, du tissu adipeux est fréquemment observé à proximité des cancers invasifs, en particulier dans le cancer du sein [16] $(\rightarrow)$ Les adipocytes sécrètent des $(\rightarrow)$ Voir la Synthèse de V. Laurent et al., molécules pro-inflammatoires, page 398 de ce numéro des molécules qui favorisent le remodelage de la matrice extracellulaire et libèrent des acides gras qui facilitent la croissance tumorale. Ces interactions constituent l'une des bases de l'augmentation de l'incidence et de l'agressivité des cancers chez les obèses. Les maladies inflammatoires du côlon (maladie de Crohn, rectocolite hémorragique, etc.), du poumon (bronchite chronique, silicose, etc.), de la plèvre (asbestose), du foie (cirrhoses alcooliques ou virales) sont elles aussi associées à une incidence accrue de cancers dans leurs organes respectifs et considérées comme des états précancéreux. La prise d'aspirine ou d'autres anti-inflammatoires a d'ailleurs montré une certaine efficacité dans la prévention des cancers du côlon.

\section{La transition tumeur précancéreuse-tumeur invasive}

Sur un terrain d'inflammation chronique et de forte vascularisation, des cellules ayant subi des premières modifications précancéreuses se multiplient et peuvent évoluer d'un état de dysplasie vers une tumeur bien encapsulée, souvent appelée carcinome in situ. L'ablation de la tumeur à ce stade est souvent curative mais, si elle n'est pas faite, la tumeur peut évoluer vers un cancer localement invasif.

Les cellules immunitaires pénètrent dans les tumeurs solides par extravasation. Le réseau vasculaire apporte 


\begin{tabular}{|c|c|}
\hline Agents physiques & Cancers associés \\
\hline Rayons X & Leucémies, lymphomes \\
\hline UV & Mélanomes \\
\hline \multirow{2}{*}{ Radionucléides } & Cancer de la thyroïde \\
\hline & Leucémies \\
\hline \multicolumn{2}{|l|}{ Agents chimiques } \\
\hline \multirow{3}{*}{ Tabac } & Cancer du poumon \\
\hline & Cancer ORL \\
\hline & Cancer de la vessie \\
\hline \multirow{2}{*}{ Amiante } & Mésothéliome \\
\hline & Cancer du poumon \\
\hline Goudron & Cancer des testicules \\
\hline Sciure de bois & Cancer des fosses nasales \\
\hline \multicolumn{2}{|l|}{ Agents biologiques } \\
\hline \multicolumn{2}{|l|}{ Virus } \\
\hline \multirow{3}{*}{ Virus de papillome humain } & Cancer du col de l'utérus \\
\hline & Cancer ORL \\
\hline & Cancer du rectum, cancer du vagin \\
\hline Virus des hépatites $B$ et $C$ & Cancer du foie \\
\hline Virus d'Epstein-Barr & Lymphomes \\
\hline Virus Herpès humain de type 8 & Sarcome de Kaposi \\
\hline $\begin{array}{l}\text { Virus lymphotropique T } \\
\text { humain de type } 1\end{array}$ & Lymphomes T \\
\hline \multicolumn{2}{|l|}{ Bactéries } \\
\hline Helicobacter pylori & Cancer de l'estomac \\
\hline Salmonella typhi & Cancer de la vésicule biliaire \\
\hline Chlamydia pneumoniae & Cancer du poumon \\
\hline \multicolumn{2}{|l|}{ Parasites } \\
\hline Schistosoma haematobium & Cancer de la vessie \\
\hline Opisthorchis viverrini & Cholangiocarcinome hépatique \\
\hline
\end{tabular}

Tableau I. Agents favorisant le développement d'un cancer.

également les nutriments et l'oxygène nécessaires à la multiplication des cellules tumorales. Les cellules vasculaires prolifèrent au contact de ces dernières et s'agencent en vaisseaux tumoraux de manière anarchique $[17,18](\rightarrow)$

$(\rightarrow)$ Voir les Synthèses de S. Azzi et J. Gavard, et de S. Garrido-Urbani et al., pages 408 et 415 de ce numéro

Durant cette période, qui peut durer des années, un combat s'engage entre la tumeur et une réaction immunitaire locale. Un bon exemple de l'évolution de la réponse immunitaire locale est celui des dysplasies de haut grade et des carcinomes in situ du col de l'utérus. Ces tumeurs sont la conséquence d'une infection par des formes particulières de papillomavirus (HPV16 et HPV18) [19]. Elles expriment des antigènes associés à la tumeur, codés par des gènes viraux, qui induisent une réaction immunitaire locale. Aussi longtemps que cette dernière est de type Thl avec une forte production d'IFN $\gamma$ (interféron) et de cellules T $\mathrm{CD} 8^{+}$cytotoxiques, la tumeur est contenue. Cependant, sous l'influence de molécules produites par les cellules transformées, la réaction peut être déviée vers une réaction immunitaire de type Th2 et suppressive, accompagnée d'un renforcement de la réaction inflammatoire chronique, en particulier sous I'influence de l'IL6 [20] $(\rightarrow)$

$(\rightarrow)$ Voir la Synthèse de M. Bruchard et $F$. Ghiringhelli, La tumeur devient alors un cancer page 429 de ce numéro invasif [1]. Ces états précancéreux sont des cibles idéales pour des approches vaccinales conçues pour prévenir l'évolution vers le cancer [2, 19].

\section{Le champ de bataille de la tumeur primitive}

Lorsqu'un cancer devient cliniquement détectable, il est déjà le produit d'interactions longues avec son microenvironnement qui vont marquer toute l'histoire du patient et de sa maladie. Une forte réaction immunitaire Thl/CD8 cytotoxique mémoire contrôle la tumeur primitive, freine sa progression et son invasivité loco-régionale et l'essaimage métastatique. L'identification des caractéristiques de cette réaction bénéfique aux patients, particulièrement étudiée dans le cancer colorectal et qui est aussi la règle dans de nombreux cancers, a des impacts cliniques majeurs. Elle a permis d'établir un « immunoscore » qui représente le plus fort facteur pronostique prédisant la survie des patients [21] $(\rightarrow)$, et elle représente l'objectif à atteindre pour les traitements immunomodulateurs.

$\rightarrow$ Voir la Synthèse de J. Galon et al., page 439 de ce numéro L'analyse de nombreuses tumeurs a aussi permis d'identifier des structures lymphoïdes proches des massifs tumoraux et qui pourraient être un lieu de génération des réponses immunes antitumorales [3]. En effet, ces structures lymphoïdes sont composées d'une zone $T$ et d'une zone $B$. La zone T contient des cellules dendritiques matures au contact de lymphocytes T CD4 $4^{+}$et $\operatorname{CD} 8^{+}$. C'est le seul endroit de la tumeur où résident des lymphocytes $T$ naïfs qui arrivent par des high endothelial venules (veinules à endothélium épais) et seront potentiellement activés et éduqués par les cellules dendritiques matures au sein des tumeurs. Les lymphocytes B prolifèrent et produisent des anticorps. II s'agit donc de véritables structures lymphoïdes tertiaires, induites par la tumeur 


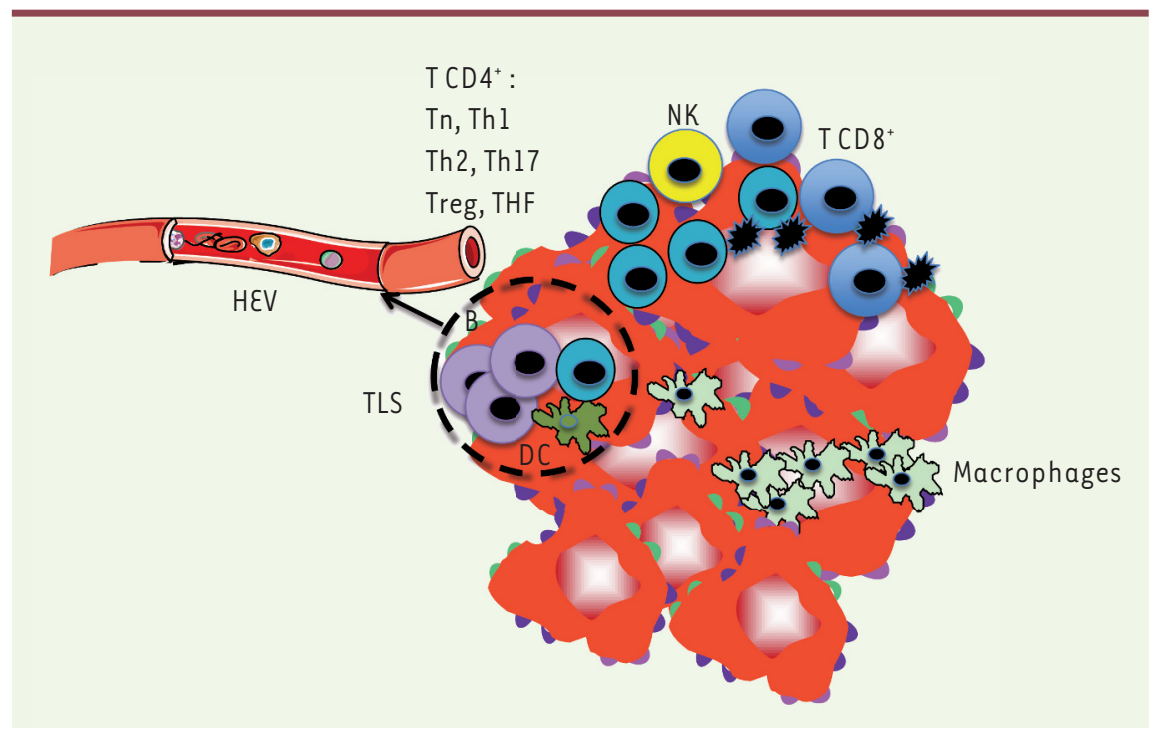

Figure 1. Le microenvironnement immunitaire des tumeurs. Les tumeurs contiennent tous les éléments du système immunitaire: lymphocytes (T, B, NK [natural killer]), cellules dendritiques, macrophages et autres cellules myéloïdes) qui pénètrent à partir des vaisseaux. Des mini-organes lymphoïdes (TLS) qui contiennent des cellules dendritiques, des lymphocytes $T$ et des lymphocytes $B$ se développent dans le stroma tumoral, permettant la génération d'une réponse immunitaire locale qui va s'opposer à l'invasion tumorale et aux métastases, et qui est effectuée notamment grâce aux lymphocytes $T$ effecteurs Thl et T mémoires. Tn : lymphocytes T naïfs.

(on ne les trouve pas à distance dans le tissu sain) par le biais de chimiokines et de cytokines produites par des cellules tumorales et du microenvironnement [4-6]. Elles ressemblent aux structures lymphoïdes secondaires des ganglions réactionnels et aux structures lymphoïdes tertiaires trouvées aux sites inflammatoires des maladies auto-immunes [22], des sites infectés ou en cours d'un rejet de greffe [7]. La densité de ces structures lymphoïdes tertiaires tumorales est associée à un pronostic favorable dans la majorité des cancers, et est un marqueur d'une immunothérapie efficace. Nous avons formulé l'hypothèse selon laquelle elles représentaient le lieu de génération d'une réponse immunitaire antitumorale qui permettrait de contrôler localement une tumeur primitive, et qui engendrerait des lymphocytes T mémoire centrale pouvant contenir des cellules métastatiques qui essaiment de la tumeur primitive (Figure 1, architecture immunitaire d'une tumeur).

Au cours de la progression tumorale, le paysage immunitaire se modifie : les cellules $C D 8^{+}$diminuent et les macrophages et les cellules myéloïdes suppressives augmentent $[20](\rightarrow)$.

Cette dynamique, qui résulte de facteurs tumoraux et du patient, est un facteur majeur de contrôle ou d'échappement des cancers dont l'impact sur $\rightarrow$ Voir la Synthèse de M. Bruchard et F. Ghiringhelli, page 429 de ce numéro l'espérance de vie des patients est primordial [8]. Cette dynamique peut être modifiée favorablement par les immunothérapies ciblant le microenvironnement tumoral.

\section{Les métastases : échappement irrémédiable ou tumeurs encore contrôlables ?}

Dans sa célèbre revue de 2001, R.D. Schreiber propose la théorie des $3 \varepsilon$ (élimination, équilibre, échappement) [9] comme version revisitée de la théorie de la surveillance immunitaire postulée un demi-siècle plus tôt indépendamment par L. Thomas [10] et F. Mc Farlane Burnett [11]. La théorie des $3 \varepsilon$ pose le principe de trois phases dans les relations entre les cellules tumorales et leur microenvironnement immunitaire. Lors de la première phase d'élimination, les produits des modifications oncogéniques d'une tumeur naissante sont reconnus comme néoantigènes et induisent une réaction immunitaire suffisamment efficace pour éradiquer la tumeur. C'est la théorie classique de la surveillance immunitaire. Les cellules transformées non détruites à ce stade prolifèrent et forment une tumeur primitive pendant l'évolution de laquelle s'établit un équilibre avec la réaction immunitaire locale. La tumeur peut alors être contrôlée et maintenue à son site d'origine pendant des années. Cependant, du fait de la plasticité de leur génome, les cellules tumorales peuvent échapper au contrôle immunitaire, croître et disséminer. Dans cette hypothèse, les sites métastatiques d'un cancer devraient présenter un microenvironnement radicalement différent de celui du site primitif sans véritable contrainte sur leur croissance. Des travaux très récents ont remis cette hypothèse en question. L'analyse comparée des microenvironnements des cancers colorectaux et rénaux primitifs et de leurs métastases pulmonaires respectives a révélé que les organisations vasculaires, inflammatoires et immunitaires des sites métastatiques étaient du même type que ceux de la tumeur d'origine. Ainsi, les métastases pulmonaires des cancers colorectaux sont peu vascularisées, relativement peu inflammatoires et riches en structures lymphoïdes tertiaires, comme la tumeur primitive. De plus, lorsque la densité de lymphocytes $T$, en particulier $\mathrm{CD}^{+}$, est forte dans la tumeur primitive, il en est de même dans les sites métastatiques même s'ils sont détectés des années plus tard. Enfin, l'impact pronostique favorable d'un immunoscore élevé au site primitif s'applique aussi au niveau des métastases. À l'opposé, les métastases pulmonaires de cancer rénal sont fortement vascularisées, 


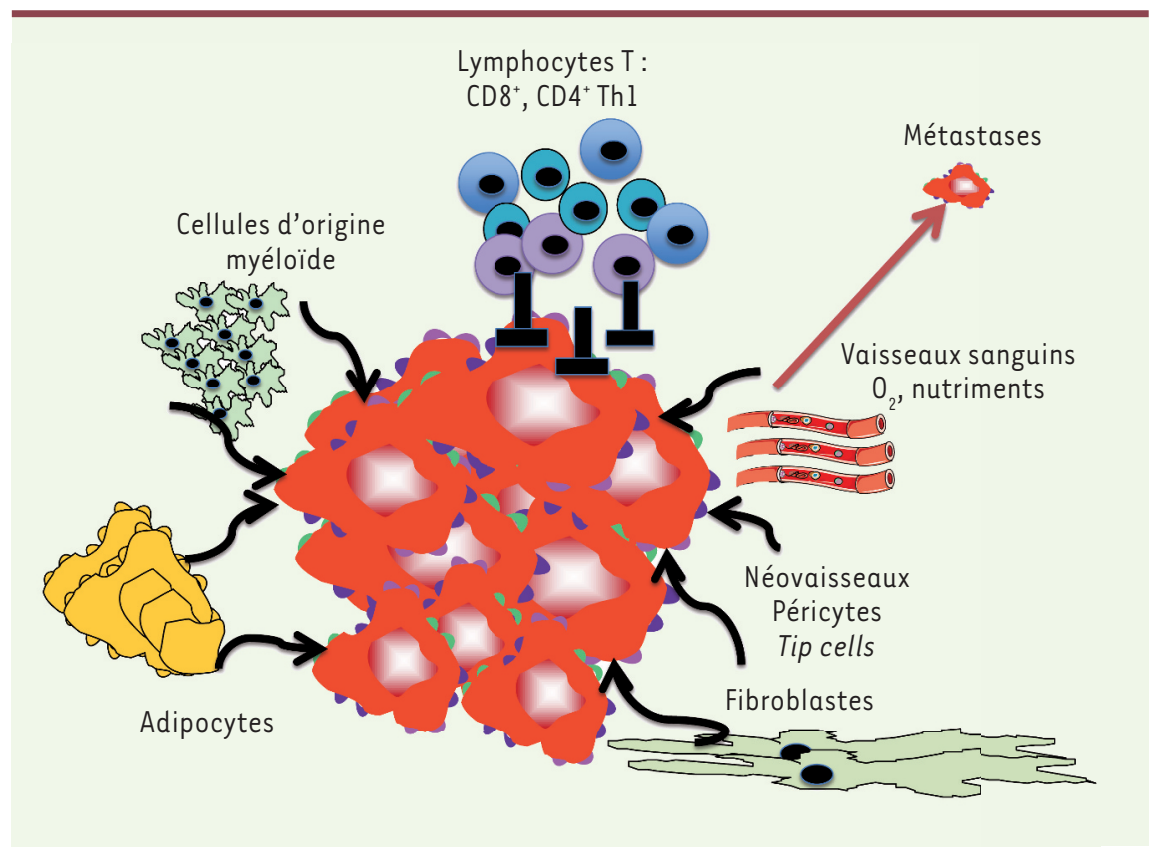

Figure 2. Interactions entre la tumeur et son environnement. Cette figure synthétise les influences entre la tumeur et le microenvironnement décrites dans les articles de ce numéro. Les tissus sont profondément modifiés par l'existence d'une tumeur en croissance, le stroma tumoral, les fibroblastes, les vaisseaux sanguins et lymphatiques, les néovaisseaux, réagissent à la tumeur et contribuent à son développement (flèches noires). Il est frappant de constater que parmi tous les éléments du microenvironnement tumoral, les lymphocytes $\operatorname{TCD} 8^{+}$et les lymphocytes $C D 4^{+} T h l$ générés par la réponse immunitaire adaptative ont une valeur pronostique positive sur la survie des patients dans la majorité des cancers (barres noires). Les autres catégories de lymphocytes infiltrants (Treg, Th17, TFH

[follicular helper $T$ cells], B) ont, soit une valeur pronostique qui reste à déterminer sur de grandes séries, soit une valeur pronostique qui dépend du type de cancer et de son environnement.

très inflammatoires et pauvres en structures lymphoïdes tertiaires, comme les tumeurs rénales primitives. Là encore, on retrouve une conservation entre le microenvironnement d'une tumeur chez un patient et celui des différents sites où elle essaime. Une donnée supplémentaire a révélé que même chez les patients présentant une forte infiltration de lymphocytes $T$ CD $8^{+}$dans leur tumeur primitive et ses métastases, celle-ci est associée à un pronostic défavorable en raison du contexte vasculaire et inflammatoire de ces tumeurs. L'ensemble de ces données, confirmées par l'analyse d'autres sites métastatiques comme le foie pour les cancers colorectaux, permettent d'établir plusieurs points essentiels. (1) À la phase métastatique, la tumeur n'échappe pas au contrôle favorable ou défavorable de son microenvironnement. (2) Le dialogue établi par les cellules tumorales et leur microenvironnement immunitaire, inflammatoire, vasculaire pendant la longue période de génération et d'établissement d'un cancer, produit une empreinte qui est reproduite aux différents sites de développement de la maladie. Cette empreinte est la marque de l'histoire d'un cancer chez un patient. Les cellules immunitaires mémoires engendrées dans les structures lymphoïdes tertiaires jouent probablement un rôle majeur dans la répétition de ce dialogue aux différents sites. (3) Par les chimiokines, cytokines, facteurs de vascularisation qu'elles produisent, les cellules tumorales modulent leur microenvironnement plus fortement que ne le fait l'organe où elles se développent puisque cet environnement est similaire dans un côlon, un foie ou un poumon pour un cancer colorectal, ou encore un rein et un poumon pour un cancer rénal et ceci représente une règle générale. (4) Enfin, un ciblage thérapeutique efficace du microenvironnement tumoral est possible, et souhaitable, à tous les stades de développement d'un cancer.

\section{Traiter un cancer en ciblant son microenvironnement}

La thérapie des cancers a longtemps eu comme unique objectif l'élimination directe des cellules tumorales. Chirurgie, radiothérapie, chimiothérapies classiques veulent extirper, bloquer la prolifération ou détruire les cellules malignes. Depuis quelques années, le microenvironnement tumoral est aussi pris en considération dans le traitement des cancers [23] $(\rightarrow)$.

$(\rightarrow)$ Voir la Synthèse

de L. Borriello

et Y.A. DeClerk, page 445 de ce numéro

Selon les cancers et la structure

de leur microenvironnement, différents agents ont été développés et utilisés avec un succès grandissant pour moduler les interactions entre les cellules tumorales et leur hôte. Ainsi, les agents bloquant la vascularisation (anticorps anti-VEGF [vascular endothelial growth factor] ou inhibiteurs du récepteur du VEGF) diminuent la vascularisation tumorale. Mais, ils sont aussi des immunostimulants [12], efficaces dans le cancer du rein, en particulier. Les anticorps anti-CTLA4 (cytotoxic T-lymphocyte antigen 4) et anti PDI (programmed cell death protein 1 ), qui «déverrouillent » les réactions immunitaires et permettent un influx de lymphocytes $T$ cytotoxiques aux sites tumoraux, sont efficaces dans le mélanome [24], le cancer du rein, le cancer du poumon, même au stade métastatique. Des molécules qui diminuent l'activité des cellules suppressives, myéloïdes ou lymphocytaires sont testées. Les vaccinations contre 
1. Les chimiothérapies immunogéniques, comme l'oxaliplatine, qui induisent l'expression de calréticuline sur les cellules tumorales, ce qui augmente la phagocytose et la présentation antigénique, et aide à l'élimination des cellules tumorales par le système immunitaire adaptatif

2. Les traitements anti-angiogéniques (comme le sunitinib et le sorafenib), qui diminuent les cellules myéloïdes suppressives, les Treg circulants et intratumoraux, ce qui module l'immunité antitumorale

3. Les anticorps anti-points de contrôle immunitaires, qui ciblent par exemple CTLA4, PD1, PD1L, augmentent l'infiltrat T, TCD8 ${ }^{+}$ en particulier, ce qui augmente l'immunité antitumorale

4. Les vaccins anticancer, qui augmentent l'infiltrat $T$ dans les tumeurs, ce qui augmente la réponse cytotoxique antitumorale

5. Les anticorps monoclonaux antitumeurs, qui induisent une immunité adaptative antitumorale

Tableau II. Les traitements immunomodulateurs qui modifient le microenvironnement immunitaire des tumeurs.

des antigènes tumoraux entraînent aussi, lorsqu'elles sont efficaces comme dans certains cancers de la prostate, un afflux de lymphocytes T CD8 ${ }^{+}$aux sites tumoraux. Même des médicaments qui ciblent les cellules malignes comme les anticorps monoclonaux anti-CD20 (ciblant les lymphocytes B) ou anti-HER2 neu (human epidermal growth factor receptor 2 ), efficaces dans le traitement de lymphomes et de cancers du sein respectivement, induisent une réaction immunitaire antitumorale $T$ mémoire associé au pronostic à long terme du patient $(\rightarrow)$ [25]. Un influx de lymphocytes T CD8 ${ }^{+}$ intratumoraux a été rapporté après traitement avec des anticorps bispécifiques dont un bras cible $(\rightarrow)$ Voir la Synthèse de C. Deligne et J.L. Teillaud, $m / s n^{\circ} 1$, janvier 2013, page 57 la tumeur et l'autre les lymphocytes T. Les thérapies cellulaires utilisant des lymphocytes $T$ antitumoraux poursuivent le même objectif. Enfin, même les chimiothérapies classiques ou ciblées peuvent induire une mort immunogénique des cellules tumorales, qui stimule la réaction immunitaire locale et entraîne un contrôle à long terme du cancer. Ces différentes approches sont résumées dans le Tableau II.

\section{Conclusion}

Longtemps considéré comme une maladie génétique et cellulaire, le cancer est aujourd'hui envisagé comme une maladie de système dont l'issue dépend en grande partie des interactions de la tumeur avec l'hôte, en particulier au sein du microenvironnement tumoral. Celui-ci peut favoriser la croissance tumorale ou la contraindre. II se modifie au cours de l'évolution de la maladie et la compréhension de cette dynamique permet d'identifier de nouveaux facteurs pronostiques et de nouvelles cibles thérapeutiques aux différents stades de la maladie cancéreuse. $\diamond$

\section{LIENS D'INTÉRÊT}

Les auteurs déclarent n'avoir aucun lien d'intérêt concernant les données publiées dans cet article.

\section{SUMMARY}

The tumoral microenvironment: feeding resources, battle field and therapeutic target in cancer

Tumors grow surrounded by a complex cellular and tissular microenvironment. Its components influence disease outcome, some accelarating growth and invasion and others blocking it. From the very first steps leading a normal tissue to dysplasia, followed by a benign tumor which may become locally invasive and metastatic, interactions between the transformed cells and their microenvironment direct the evolution of this process. Chronic inflammation in a tissue favors tumor first appearance which then accentuate the nutritional and energetic properties of their microenvironment by producing neoangiogenic, immunosuppressive and inflammatory molecules. In contrast a strong memory Thl/ cytotoxic reaction can prevent the transition from benign to malignant tumors and control cancer invasivness and metastasis. Knowledge of these multiple phenomena, discussed in the different articles of the present issue has a major clinical impact, since new prognostic tests and efficient novel therapeutic approaches which target the tumor microenvironment are actively developed. $\diamond$

\section{RÉFÉRENCES}

1. Tartour E, Gey A, Sastre-Garau X, et al. Analysis of interleukin 6 gene expression in cervical neoplasia using a quantitative polymerase chain reaction assay: evidence for enhanced interleukin 6 gene expression in invasive carcinoma. Cancer Res $1994 ; 54: 6243-8$.

2. Van Poelgeest MI, Welters MJ, van Esch EM, et al. HPV16 synthetic long peptide (HPV16-SLP) vaccination therapy of patients with advanced or recurrent HPV16-induced gynecological carcinoma, a phase II trial. J Transl Med $2013 ; 11: 88$.

3. Dieu-Nosjean MC, Antoine M, Danel C, et al. Long-term survival for patients with non-small-cell lung cancer with intratumoral lymphoid structures. J Clin Oncol $2008 ; 26$ : 4410-7.

4. De Chaisemartin L, Goc J, Damotte D, et al. Characterization of chemokines and adhesion molecules associated with $\mathrm{T}$ cell presence in tertiary lymphoid structures in human lung cancer. Cancer Res $2011 ; 71: 6391-9$.

5. Goc J, Germain C, Vo-Bourgais T, et al. Tertiary lymphoid structure dendritic cells signal a Thl and cytotoxic immune contexture, and are required for good prognostic value of $\mathrm{CD} 8^{+} \mathrm{T}$ cells in lung cancer patients. Cancer Res $2014 ; 74: 705-15$

6. Fridman WH, Pagès F, Sautès-Fridman C, Galon J. The immune contexture in human tumours: impact on clinical outcome. Nat Rev Cancer 2012; 12 : 298-306.

7. Aloisi F, Pujol-Borrell R. Lymphoid neogenesis in chronic inflammatory diseases. Nat Rev Immunol 2006 ; 6 : 205-17.

8. Bindea G, Mlecnik B, Tosolini M, et al. Spatiotemporal dynamics of intratumoral immune cells reveal the immune landscape in human cancer. Immunity 2013 ; 39 : 782-95.

9. Dunn GP, Old L), Schreiber RD.The three Es of cancer immunoediting. Annu Rev Immunol $2004 ; 22: 329-60$ 


\section{RÉFÉRENCES}

10. Thomas L. Discussion. In : Lawrence HS, ed. Cellular and humoral aspects of the hypersensitive states. New York : Hoeber-Harper, 1959 : 529-32.

11. Burnet FM. Immunological aspects of malignant disease. Lancet $1967 ; 289$ : 1171-4.

12. Tartour $\varepsilon$, Pere $H$, Maillere B, et al. Angiogenesis and immunity: a bidirectional link potentially relevant for the monitoring of antiangiogenic therapy and the development of novel therapeutic combination with immunotherapy. Cancer Metastasis Rev $2011 ; 30: 83-95$.

13. Hubert S, Abastado JP. Les étapes précoces du processus métastatique. Med Sci (Paris) 2014 ; $30: 378-84$.

14. Buache $\varepsilon$, Rio MC. Le stroma tumoral, un terreau fertile pour la cellule cancéreuse. Med Sci (Paris) $2014 ; 30: 385-90$

15. Albrengues J, Meneguzzi G, Gaggioli C. L'invasion des cellules tumorales : quand les fibroblastes s'en mêlent. Med Sci (Paris) 2014 ; 30 : 391-7.

16. Laurent $\mathrm{V}$, Nieto $\mathrm{L}$, Valet $\mathrm{P}$, Muller $\mathrm{C}$. Tissu adipeux et cancer : une association à haut risque. Med Sci (Paris) $2014 ; 30: 398-404$

17. Azzi S, Gavard J. Vaisseaux sanguins et tumeurs ou l'art du dialogue. Med Sci (Paris) 2014 ; $30: 408-14$.

18. Garrido-Urbani S, Jaquet V, Imhof BA. ERO, NADPH oxydases et vascularisation des tumeurs. Med Sci (Paris) $2014 ; 30: 415-21$

19. Heard I, Floret D. Vaccins contre les papillomavirus humains (HPV). Dernières recommandations du Haut conseil de la santé publique, et premiers résultats cliniques et virologiques. Med Sci (Paris) $2013 ; 29$ : 1161-6.
20. Bruchard M, Ghiringhelli F. Microenvironnement tumoral : cellules régulatrices et cytokines immunosuppressives. Med Sci (Paris) 2014 ; $30: 429-35$.

21. Galon J, Bindea G, Mlecnik B, et al. Microenvironnement immunitaire et cancer : intérêt de l'Immunoscore pour prédire l'évolution clinique. Med Sci (Paris) $2014 ; 30: 439-44$.

22. Perros $F$, Humbert $M$, Cohen-Kaminsky $S$. Hypertension artérielle pulmonaire. Med Sci (Paris) $2013 ; 29: 607-16$

23. Borriello L, DeClerck YA. Le microenvironnement tumoral et la résistance thérapeutique. Med Sci (Paris) $2014 ; 30: 445-51$.

24. Robert C, Mateus C. Anticorps anti-CTLA-4. Une avancée thérapeutique majeure dans le traitement du mélanome métastatique. Med Sci (Paris) $2011 ; 27: 850-8$.

25. Deligne C, Teillaud JL. Le double visage des anticorps monoclonaux en oncologie. Med Sci (Paris) $2013 ; 29: 57-63$

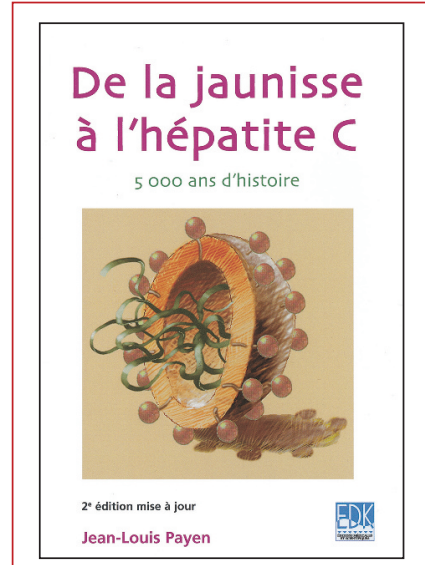

ISBN : 978-2-8425-4136-1 128 pages a jaunisse est un symptôme facilement identifiable ; il paraissait bien naturel que l'homme, confronté à une modification de la couleur de ses yeux et de sa peau ait de tous temps recherché les causes de cette transformation.

II n'est donc pas surprenant que le premier traité de médecine, écrit 3000 ans avant J.C. par un médecin sumérien, décrive déjà la jaunisse. À chaque époque de l'histoire de la médecine, les praticiens, influencés par les concepts médicaux de leur temps, attribuèrent une ou plusieurs explications particulières à ce symptôme. Ainsi, du démon Ahhâzu des Sumériens à la sophistication des biotechnologies qui permirent la découverte du virus de l'hépatite $C$, le lecteur cheminera sur une période de 5000 ans au travers des différents continents. Ici encore, l'histoire se révèle une formidable source de réflexion : le foie souvent impliqué dans l'apparition des jaunisses est-il le siège de l'âme?

Les expérimentations humaines chez des volontaires ou chez des enfants handicapés mentaux étaient-elles justifiées pour permettre la découverte des virus des hépatites?

Le formidable développement de la transfusion sanguine, des vaccinations, mais aussi de la toxicomanie explique-t-il les épidémies d'hépatites du XXe siècle?

Autant de questions qui sont abordées dans ce livre passionnant et accessible à tous.

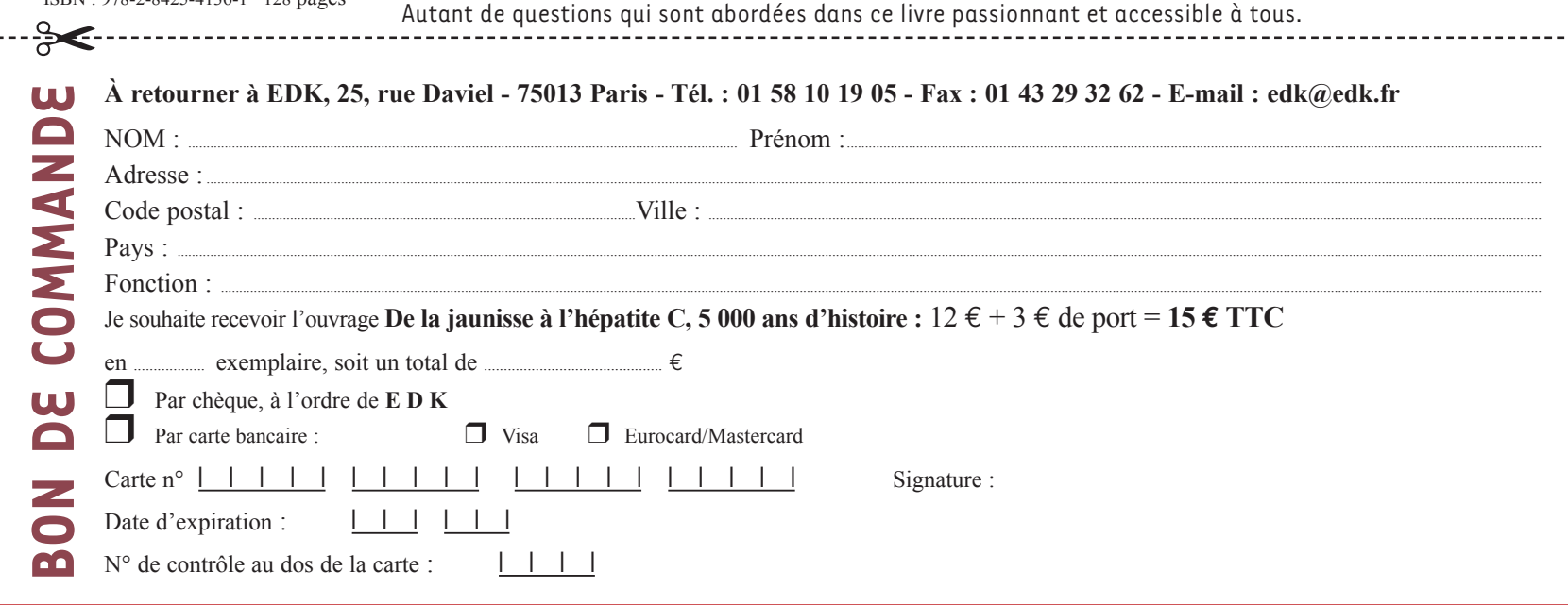

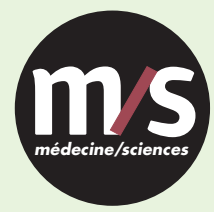

Tarifs d'abonnement $\mathrm{m} / \mathrm{s}-2014$

Abonnez-vous

à médecine/sciences
$>$ Grâce à $m / s$, vivez en direct les progrès des sciences biologiques et médicales

Bulletin d'abonnement page 470 dans ce numéro de $\mathrm{m} / \mathrm{s}$

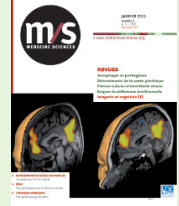

